\title{
Algebrability of some subsets of the disk algebra
}

\author{
Mary Lilian Lourenço Daniela M. Vieira
}

\begin{abstract}
We show that the subset of the disk algebra of the functions that are not in some Dales-Davie algebra is algebrable. In other words, the set $\{f \in \mathcal{A}(D)$ : $\left.\sum_{n=0}^{\infty} \frac{\left\|f^{(n)}\right\|}{n !}=+\infty\right\}$ is shown to be algebrable.
\end{abstract}

\section{Introduction}

In the last ten years, several authors have searched large algebraic structures (linear spaces or algebras) in spaces of functions enjoying a special property. If a vector space $V$ has a subset $M$ such that $M \cup\{0\}$ contains an infinite-dimensional vector space, then $M$ is called lineable. If $M \cup\{0\}$ contains a closed infinitedimensional vector space, then $M$ is called spaceable. The origin of the concept of lineability is due to Gurariy [11], see also [12], that showed that there exists an infinite dimensional linear space contained in the set of nowhere differentiable functions on $[0,1]$. Recently, Aron et al in [5] published a book dedicated to this subject. Since Gurariy's definition, it was natural to study subsets in spaces of functions which contains an infinitely generated algebra. Such spaces are called algebrable. It is clear that algebrability implies lineability. The concept of algebrability has been also defined by Gurariy and first pointed out in [6], but then has rapidly been investigated by other authors, for example [3, 4, 6, 7, 8]. In [8] Aron and Seoane-Sepúlveda showed that there exists an infinitely generated algebra in the set of everywhere surjective functions on $\mathbb{C}$. In [7], Aron, Pérez-García and Seoane-Sepúlveda showed that the set of continuous functions whose Fourier series expansion diverges is algebrable.

Received by the editors in March 2016 - In revised form in April 2016.

Communicated by F. Bastin.

2010 Mathematics Subject Classification : Primary 46E25. Secondary 15A03.

Key words and phrases : algebrability, Dales-Davie algebra, disk algebra. 
In 1973, Dales and Davie [10] introduced and studied some algebras of differentiable functions on a given perfect compact subset $X \subset \mathbb{C}$. These algebras were called Dales-Davie algebras by Abtahi and Honary in [1], and they denoted them by $\mathcal{D}(X, M)$. If $D$ denotes the open unit disk and $X=\bar{D}$, then $\mathcal{D}(\bar{D}, M)$ is a subalgebra of the disk algebra $\mathcal{A}(D)$. In this article, we study how big the difference $\mathcal{A}(D) \backslash \mathcal{D}(\bar{D}, M)$ is. Indeed, we show that the set $\mathcal{A}(D) \backslash \mathcal{D}(\bar{D}, M)$ contains a closed infinitely generated algebra, that is, it is algebrable and naturally is spaceable.

We refer the interested reader to [5,9] for a wider range of results in the topic of lineability and algebrability, and to $[1,2,10,13]$ for further informations on the Dales-Davie algebras.

\section{Preliminaries}

Let $X \subset \mathbb{C}$ be a perfect, compact plane set. A complex valued function $f: X \longrightarrow \mathbb{C}$ is differentiable at a point $z_{0} \in X$ if the following limit exists:

$$
f^{\prime}\left(z_{0}\right)=\lim \left\{\frac{f(z)-f\left(z_{0}\right)}{z-z_{0}}: z \in X, z \rightarrow z_{0}\right\} \text {. }
$$

A complex valued function $f$ is differentiable on $X$ if it is differentiable at every point of $X$. Note that, if $f$ is differentiable on $X$, it is analytic on (int) $X$. The algebra of functions on $X$ with continuous $n$-th derivative is denoted by $\mathcal{D}^{n}(X)$, and $\mathcal{D}^{\infty}(X)$ denotes the algebra of functions on $X$ with derivative of all orders. For every $f \in \mathcal{D}^{\infty}(X)$ we denote the $n$-th derivative of $f$ by $f^{(n)}$, for all $n \in \mathbb{N}$. Also, $\left\|f^{(n)}\right\|_{X}=\sup _{z \in X}\left|f^{(n)}(z)\right|$, for all $n \in \mathbb{N}$.

Let $\left(M_{n}\right)_{n \in \mathbb{N}}$ be a sequence of positive numbers such that $M_{0}=1$, and for each $n \geq 1$,

$$
\frac{M_{n}}{M_{k} M_{n-k}} \geq\left(\begin{array}{l}
n \\
k
\end{array}\right) \quad(0 \leq k \leq n) .
$$

The sequence $M=\left(M_{n}\right)_{n \in \mathbb{N}}$ is called an algebra sequence if it satisfies the above conditions.

The Dales-Davie algebras on $X$ are defined by

$$
\mathcal{D}(X, M)=\left\{f \in D^{\infty}(X): \sum_{n=0}^{\infty} \frac{\left\|f^{(n)}\right\|_{X}}{M_{n}}<+\infty\right\} .
$$

The norm on $\mathcal{D}(X, M)$ is defined by $\|f\|=\sum_{n=0}^{\infty} \frac{\left\|f^{(n)}\right\|_{X}}{M_{n}}$. These algebras were introduced and studied by Dales and Davie in [10], and they have been investigated by Abtahi and Honary in $[1,2,13]$.

For each sequence $M=\left(M_{n}\right)_{n \in \mathbb{N}}$ of positive numbers, $\mathcal{D}(X, M)$ is a normed vector space. When $M=\left(M_{n}\right)_{n \in \mathbb{N}}$ is an algebra sequence, then $\mathcal{D}(X, M)$ is a normed algebra.

Let $\mathcal{B}$ be an algebra over $\mathbb{K}=\mathbb{R}$ or $\mathbb{C}$. In this paper, the dimension of $\mathcal{B}$, denoted by $\operatorname{dim}(\mathcal{B})$, will always refer to its dimension as a vector space. Let 
$S=\left\{z_{i}: i \in I\right\}$ be a subset of an algebra $\mathcal{B}$. The algebra generated by $S$ is the set

$$
\mathcal{A}(S)=\left\{\sum_{j=1}^{k} \alpha_{j} z_{i}^{j}, \alpha_{j} \in \mathbb{K}, z_{i} \in S, i \in I, k \in \mathbb{N}\right\} .
$$

The set $S$ is called a system of generators of $\mathcal{A}(S)$. A system of generators $S$ is minimal if for every $i_{0} \in I, z_{i_{0}} \notin \mathcal{A}\left(S \backslash\left\{z_{i_{0}}\right\}\right)$. As usual, we denote by $\mathbb{C}\left[z_{1}, z_{2}\right]$ the ring of all polynomials in two complex variables. We say that two elements $a$ and $b$ of an algebra $\mathcal{B}$ are algebraically independent if $P \in \mathbb{C}\left[z_{1}, z_{2}\right]$ is such that $P(a, b)=0$ then $P \equiv 0$.

Definition 2.1. Let $A$ be a subset of an algebra. We say that $A$ is

(a) lineable if $A \cup\{0\}$ contains an infinite dimensional vector space.

(b) spaceable if $A \cup\{0\}$ contains a closed infinite dimensional vector space.

(c) algebrable if there is an algebra $\mathcal{B} \subset A \cup\{0\}$, such that $\mathcal{B}$ has an infinite minimal system of generators.

(d) $(\alpha, \beta)$-algebrable if there is an algebra $\mathcal{B} \subset A \cup\{0\}$, with $\operatorname{dim}(\mathcal{B})=\alpha$ and $\operatorname{card}(S)=\beta$, where $\alpha$ and $\beta$ are two cardinal numbers, and $S$ is a minimal system of generators of $\mathcal{B}$.

Other concepts such as maximal lineability, dense-lineability, spaceabilily, etc can be found in [9].

Let $D$ denote the open unit disk of the complex plane, that is, $D=\{z \in \mathbb{C}$ : $|z|<1\}$. The Banach algebra of continuous functions on $\bar{D}$ and analytic on $D$ with the sup norm is denoted by $\mathcal{A}(D)$. As usual we call $\mathcal{A}(D)$ the disk algebra. When $X=\bar{D}$ it follows that $\mathcal{D}(\bar{D}, M)$ is a subalgebra of $\mathcal{A}(D)$. However, the difference $\mathcal{A}(D) \backslash \mathcal{D}(\bar{D}, M)$ is not a vector space, hence not an algebra.

In this article, we want to investigate the algebrability of the set $\mathcal{A}(D) \backslash \mathcal{D}(\bar{D}, M)$. We will prove that $\mathcal{A}(D) \backslash \mathcal{D}(\bar{D}, M)$ contains an infinitely generated algebra, for several algebra sequences $M=\left(M_{n}\right)_{n \in \mathbb{N}}$.

For a fixed algebra sequence $\left(M_{n}\right)_{n \in \mathbb{N}}$, we denote

$$
\mathcal{H}(M)=\left\{f \in \mathcal{A}(D): \sum_{n=0}^{\infty} \frac{\left\|f^{(n)}\right\|_{X}}{M_{n}}=+\infty\right\} .
$$

When $M_{n}=n$ !, for all $n \in \mathbb{N}$, we will write $\mathcal{H}$ instead of $\mathcal{H}(M)$.

We show in the next Lemma that the set $\mathcal{H}$ is nonempty.

Lemma 2.2. Let $w=2 e^{i \theta}$, where $0 \leq \theta<2 \pi$. Let $f(z)=\frac{1}{z-w}$, for all $z \in \bar{D}$. Then $f \in \mathcal{H}$.

Proof. It is clear that $f \in \mathcal{A}(D)$ and that $\|f\|=\left|f\left(e^{i \theta}\right)\right|=1$. It is also easy to see that

$$
f^{(n)}(z)=\frac{(-1)^{n} n !}{(z-w)^{n+1}}=(-1)^{n} n !(f(z))^{n+1}, \forall z \in \bar{D} .
$$


Therefore $\left\|f^{(n)}\right\|=n !\|f\|^{n+1}=n !$. Then

$$
\sum_{n=0}^{\infty} \frac{\left\|f^{(n)}\right\|}{n !}=+\infty
$$

In Section 3, we will show that $\mathcal{H}$ is algebrable. Actually, we show that $\mathcal{H} \cup\{0\}$ contains a closed infinitely generated algebra, and as a consequence it is spaceable. However, it is possible to prove that $\mathcal{H}$ is spaceable with a different technique. Also, we get that $\mathcal{H}$ is $\left(\aleph_{0}, 1\right)$-algebrable. These results will be presented in the sequel.

Proposition 2.3. The set $\mathcal{H}$ is spaceable.

Proof: Let $f(z)=\frac{1}{z-2}$. Then $f \in \mathcal{H}$ by Lemma 2.2. Let $S=\left\{f^{n}: n \in \mathbb{N}\right.$, $n \geq 1\}$. Firstly, we will show that $S$ is a linearly independent subset of $\mathcal{H} \cup\{0\}$. So let $\beta_{1}, \beta_{2}, \cdots, \beta_{k} \in \mathbb{C}$ and suppose that $g=\sum_{j=1}^{k} \beta_{j} f^{j}=0$. For any $k$ distinct real numbers $x_{1}, x_{2}, \cdots, x_{k} \in D$ we have that $f\left(x_{1}\right), f\left(x_{2}\right), \cdots, f\left(x_{k}\right)$ are also $k$ distinct non-zero real numbers. Then we have the following system:

$$
\sum_{j=1}^{k} \beta_{j} f^{j}\left(x_{i}\right)=0, \text { for } i=1, \cdots, k
$$

Using a matrix notation, we have

$$
\left(\begin{array}{cccc}
f\left(x_{1}\right) & f\left(x_{1}\right)^{2} & \cdots & f\left(x_{1}\right)^{k} \\
f\left(x_{2}\right) & f\left(x_{2}\right)^{2} & \cdots & f\left(x_{2}\right)^{k} \\
\vdots & \vdots & \cdots & \vdots \\
f\left(x_{k}\right) & f\left(x_{k}\right)^{2} & \cdots & f\left(x_{k}\right)^{k}
\end{array}\right) \cdot\left(\begin{array}{c}
\beta_{1} \\
\beta_{2} \\
\vdots \\
\beta_{k}
\end{array}\right)=\left(\begin{array}{c}
0 \\
0 \\
\vdots \\
0
\end{array}\right)
$$

This matrix is a variation of the Vandermonde matrix, and its determinant equals to

$$
f\left(x_{1}\right) f\left(x_{2}\right) \cdots f\left(x_{k}\right) \cdot \prod_{1 \leq i<j \leq k}\left(f\left(x_{j}\right)-f\left(x_{i}\right)\right) .
$$

In our setting, this determinant is never zero, and then $\beta_{1}=\beta_{2}=\cdots=\beta_{k}=0$ showing that $S$ is a linearly independent set.

Next, we will show that the vector space generated by $S$, which will be denoted by $[S]$, is contained in $\mathcal{H} \cup\{0\}$. So let $g \in[S] \backslash\{0\}$. Then there are $\beta_{1}, \beta_{2}, \cdots, \beta_{k} \in \mathbb{C}$, not all zero, such that $g=\sum_{j=1}^{k} \beta_{j} f^{j}$. We want to show that $g \in \mathcal{H}$, which means $\sum_{n=0}^{\infty} \frac{\left\|g^{(n)}\right\|}{n !}=+\infty$. Note that

$$
\left(f^{j}\right)^{(n)}=(-1)^{n} j \cdot(j+1) \cdots(j+n-1) f^{j+n} .
$$

Since $f(1)=-1$, it follows that

$$
g^{(n)}(1)=\sum_{j=1}^{k} \beta_{j}(-1)^{n} j \cdot(j+1) \cdots(j+n-1)(-1)^{j+n}=\sum_{j=1}^{k} \beta_{j}(-1)^{j} \frac{(j+n-1) !}{(j-1) !}
$$


Then

$$
\begin{gathered}
\frac{g^{(n)}(1)}{n !}=-\beta_{1} \frac{1}{0 !}+\beta_{2} \frac{n+1}{1 !}-\beta_{3} \frac{(n+1)(n+2)}{2 !}+\cdots+ \\
+\cdots+(-1)^{k} \beta_{k} \frac{(n+1)(n+2) \cdots(n+k-1)}{(k-1) !}
\end{gathered}
$$

If the series $\sum_{n=0}^{\infty} \frac{\left|g^{(n)}(1)\right|}{n !}$ converges, then $\lim _{n \rightarrow \infty} \frac{\left|g^{(n)}(1)\right|}{n !}=0$. But it happens if, and only if, $\beta_{1}=\beta_{2}=\cdots=\beta_{k}=0$, which is not true. Then it follows that

$$
\sum_{n=0}^{\infty} \frac{\left\|g^{(n)}\right\|}{n !} \geq \sum_{n=0}^{\infty} \frac{\left|g^{(n)}(1)\right|}{n !}=+\infty .
$$

So we have proved that $\mathcal{H} \cup\{0\}$ is lineable. Now, since $\mathcal{H}$ with the topology induced by $\mathcal{A}(D)$ is closed, it follows that $\mathcal{H}$ is spaceable.

Corollary 2.4. The set $\mathcal{H}$ is $\left(\aleph_{0}, 1\right)$-algebrable.

Proof: Using the same notation of Proposition 2.3, we have that the algebra generated by $S$ equals the vector space generated by $S$.

\section{Algebrability of $\mathcal{H}(M)$}

We observe here that in the previous section, when we showed that $\mathcal{H}$ is spaceable, indeed we proved that $\mathcal{H} \cup\{0\}$ contains a closed algebra generated by a single element. Now, using a different technique, we will show that there is a closed infinitely generated algebra contained in $\mathcal{H} \cup\{0\}$, and in particular $\mathcal{H}$ is algebrable.

We will present some auxiliary results in the sequel.

Lemma 3.1. Let $\beta$ be a positive real number. Let $n \in \mathbb{N}$ and for each $r \in \mathbb{N}$ such that $1 \leq r \leq n$, consider

$$
a_{r}=\frac{1}{r !} \sum_{s=1}^{r}\left(\begin{array}{l}
r \\
s
\end{array}\right)(-1)^{r-s+n} s \cdot(s+1) \cdots(s+n-1) .
$$

Then:

(a) If $K$ is a fixed natural number, $1 \leq r \leq K$ and $n$ is even, then $\lim _{n \rightarrow \infty} \beta^{r} \frac{a_{r}}{n !}=+\infty$;

(b) $\lim _{n \rightarrow \infty} \lim _{r \rightarrow n} \beta^{r} \frac{a_{r}}{n !}=0$.

Proof. Developing the expression for $\frac{a_{r}}{n !}$, we have that:

$$
\begin{aligned}
& \frac{a_{r}}{n !}=\sum_{s=1}^{r}\left(\begin{array}{l}
r \\
s
\end{array}\right)(-1)^{r-s+n} \frac{s \cdot(s+1) \cdots(s+n-1)}{n ! r !}= \\
& \sum_{s=1}^{r}(-1)^{r-s+n} \frac{(s+n-1) !}{(r-s) ! s !(s-1) ! n !}=\sum_{s=1}^{r}(-1)^{r-s+n} \frac{(n+1)(n+2) \cdots(n+s-1)}{(r-s) ! s !(s-1) !} .
\end{aligned}
$$


(a) Note that each term of the summation above is a polynomial function defined in $\mathbb{N}$, with degree $s-1$. Then $\frac{a_{r}}{n !}$ is a polynomial function defined in $\mathbb{N}$, with degree at most $r-1$. If $n$ is even, the coefficient of the highest degree is positive.

Now, for a fixed natural number $K, 1 \leq r \leq K$ and $n$ even, it follows that $\lim _{n \rightarrow \infty} \beta^{r} \frac{a_{r}}{n !}=+\infty$.

(b)

$$
\begin{gathered}
\lim _{r \rightarrow n} \lim _{s \rightarrow r} \beta^{r}(-1)^{r-s+n} \frac{(s+n-1) !}{(r-s) ! s !(s-1) ! n !}= \\
=\lim _{r \rightarrow n} \beta^{r}(-1)^{n} \frac{(r+n-1) !}{r !(r-1) ! n !}=\beta^{n}(-1)^{n} \frac{(2 n-1) !}{n ! n !(n-1) !} .
\end{gathered}
$$

And by D'Alembert's criterion we have that

$$
\lim _{n \rightarrow \infty} \beta^{n}(-1)^{n} \frac{(2 n-1) !}{n ! n !(n-1) !}=0 .
$$

In this work we will have to deal with $n$-th order derivative of composed functions. A formula to this derivative is known as Faá di Bruno formula, but there are several variations of this formula. Here we will use the Hoppe's Formula for the $n$-th order derivative of composed functions. For better comprehension of the reader we spell out the formula here. For complete information about it we suggest [14].

Theorem 3.2. [14, Hoppe's Formula] Let $f$ and $g$ be functions with a sufficient number of derivatives, then

$$
(g \circ f)^{(n)}(z)=\sum_{r=1}^{n} \frac{g^{(r)}(f(z))}{r !} \sum_{s=0}^{r}\left(\begin{array}{l}
r \\
s
\end{array}\right)(-f(z))^{r-s}\left(f^{s}\right)^{(n)}(z) .
$$

In order to show that $\mathcal{H}$ is algebrable, we have to exhibit an infinitely generated algebra contained in $\mathcal{H}$. Since $\mathbb{C}\left[z_{1}, z_{2}\right]$ contains infinitely generated algebras, we will use $\mathbb{C}\left[z_{1}, z_{2}\right]$ as a model to construct infinitely generated algebras in $\mathcal{H}$. If $g_{1}$ and $g_{2}$ are two algebraically independent functions, then the algebra generated by them is isomorphic to $\mathbb{C}\left[z_{1}, z_{2}\right]$. Therefore $A\left(\left\{g_{1}, g_{2}\right\}\right)$ also contains infinitely generated algebras.

Our next result will allow us to apply these remarks in the main result.

Theorem 3.3. Let $g_{1}(z)=e^{z}$ and $g_{2}(z)=e^{\beta z}$, for all $z \in \mathbb{C}$, where $\beta$ is an irrational number. Let $P \in \mathbb{C}\left[z_{1}, z_{2}\right]$ be a non-constant polynomial, let $f(z)=\frac{1}{z+2}$ and consider $h=P\left(g_{1}, g_{2}\right)$. Then $h \circ f \in \mathcal{H} \cup\{0\}$.

Proof: By Lemma 2.2 we have that $f \in \mathcal{H}$ and $\|f\|=1$. If we consider $h=P\left(g_{1}, g_{2}\right)$ then there are $\alpha_{j k} \in \mathbb{C}$ such that

$$
h(z)=\sum_{j, k=0}^{m} \alpha_{j k} g_{1}^{j} g_{2}^{k}=\sum_{j, k=0}^{m} \alpha_{j k} e^{(j+\beta k) z} .
$$


If we denote $\beta_{j k}=j+\beta k$, then we have that

$$
h(f(z))=\sum_{j, k=0}^{m} \alpha_{j k} e^{\beta_{j k} f(z)}
$$

We want to show that $\sum_{n=0}^{\infty} \frac{\left\|(h \circ f)^{(n)}\right\|}{n !}=+\infty$. By Hoppe's formula (Theorem 3.2), we have that

$$
(h \circ f)^{(n)}(z)=\sum_{r=1}^{n} \frac{h^{(r)}(f(z))}{r !} \sum_{s=1}^{r}\left(\begin{array}{l}
r \\
s
\end{array}\right)(-f(z))^{r-s}\left(f^{s}\right)^{(n)} .
$$

Since $\left(f^{s}\right)^{(n)}=(-1)^{n} s \cdot(s+1) \cdots(s+n-1) f^{s+n}$, it follows that $(h \circ f)^{(n)}(z)=$

$$
=\sum_{r=1}^{n} \frac{h^{(r)}(f(z))}{r !} \sum_{s=1}^{r}\left(\begin{array}{l}
r \\
s
\end{array}\right)(-f(z))^{r-s}(-1)^{n} s \cdot(s+1) \cdots(s+n-1)(f(z))^{s+n} .
$$

Now

$$
(h \circ f)^{(n)}(-1)=\sum_{r=1}^{n} \frac{h^{(r)}(1)}{r !} \sum_{s=1}^{r}\left(\begin{array}{l}
r \\
s
\end{array}\right)(-1)^{r-s+n} s \cdot(s+1) \cdots(s+n-1) .
$$

Since

$$
h^{(r)}(1)=\sum_{j, k=0}^{m} \alpha_{j k} e^{\beta_{j k}} \beta_{j k}^{r}
$$

and

$$
a_{r}=\frac{1}{r !} \sum_{s=1}^{r}\left(\begin{array}{l}
r \\
s
\end{array}\right)(-1)^{r-s+n} s \cdot(s+1) \cdots(s+n-1),
$$

we have that

$$
(h \circ f)^{(n)}(-1)=\sum_{r=1}^{n} \sum_{j, k=0}^{m} a_{r} \alpha_{j k} e^{\beta_{j k}} \beta_{j k}^{r}
$$

We claim that $\sum_{n=0}^{\infty} \frac{\left|(h \circ f)^{(n)}(-1)\right|}{n !}=+\infty$. In this case, we have that

$$
\sum_{n=0}^{\infty} \frac{\left\|(h \circ f)^{(n)}\right\|}{n !}>\sum_{n=0}^{\infty} \frac{\left|(h \circ f)^{(n)}(-1)\right|}{n !}=+\infty,
$$

and the result follows.

To show the claim, and for a better comprehension of the next arguments, we will study the case for $m=2$, which simplifies the notation. In this case, the expression of $H_{n}$ is:

$$
\begin{aligned}
& H_{n}:=\frac{(h \circ f)^{(n)}(-1)}{n !}= \\
& =\sum_{r=1}^{n} \frac{a_{r}}{n !}\left(\alpha_{01} e^{\beta_{01}} \beta_{01}^{r}+\alpha_{02} e^{\beta_{02}} \beta_{02}^{r}+\cdots+\alpha_{22} e^{\beta_{22}} \beta_{22}^{r}\right)=
\end{aligned}
$$




$$
\begin{aligned}
= & \frac{a_{1}}{n !}\left(\alpha_{01} e^{\beta_{01}} \beta_{01}+\alpha_{02} e^{\beta_{02}} \beta_{02}+\cdots+\alpha_{22} e^{\beta_{22}} \beta_{22}\right)+ \\
& +\frac{a_{2}}{n !}\left(\alpha_{01} e^{\beta_{01}} \beta_{01}^{2}+\alpha_{02} e^{\beta_{02}} \beta_{02}^{2}+\cdots+\alpha_{22} e^{\beta_{22}} \beta_{22}^{2}\right)+\ldots \\
& +\frac{a_{n}}{n !}\left(\alpha_{01} e^{\beta_{01}} \beta_{01}^{n}+\alpha_{10} e^{\beta_{10}} \beta_{10}^{n}+\cdots+\alpha_{22} e^{\beta_{22}} \beta_{22}^{n}\right) .
\end{aligned}
$$

If we consider

$$
L_{r}=\sum_{j, k=0}^{2} \alpha_{j k} e^{\beta_{j k}} \beta_{j k}^{r}, \text { for all } r \in \mathbb{N},
$$

then

$$
H_{n}=\sum_{r=1}^{n} \frac{a_{r}}{n !} L_{r}
$$

Observe that $m=2$ implies only the number of terms of $L_{r}$.

We claim that there exists $N \in \mathbb{N}$ such that $L_{N} \neq 0$. Indeed, suppose that $L_{r}=0$, for all $r \in \mathbb{N}$. In particular, we have that $L_{1}=L_{2}=\cdots=L_{8}=0$. That is:

$$
\sum_{j, k=0}^{2} \alpha_{j k} e^{\beta_{j k}} \beta_{j k}^{r}=0, \text { for each } 1 \leq r \leq 8 .
$$

That means that the following system holds.

$$
\left(\begin{array}{cccc}
e^{\beta_{01}} \beta_{01} & e^{\beta_{02}} \beta_{02} & \cdots & e^{\beta_{22}} \beta_{22} \\
e^{\beta_{01}} \beta_{01}^{2} & e^{\beta_{02}} \beta_{02}^{2} & \cdots & e^{\beta_{22}} \beta_{22}^{2} \\
\vdots & \vdots & \cdots & \vdots \\
e^{\beta_{01}} \beta_{01}^{8} & e^{\beta_{02}} \beta_{02}^{8} & \cdots & e^{\beta_{22}} \beta_{22}^{8}
\end{array}\right) \cdot\left(\begin{array}{c}
\alpha_{01} \\
\alpha_{02} \\
\vdots \\
\alpha_{22}
\end{array}\right)=\left(\begin{array}{c}
0 \\
0 \\
\vdots \\
0
\end{array}\right) .
$$

The determinant of the matrix above is $\prod e^{\beta_{j k}} \beta_{j k}\left(\beta_{j k}-\beta_{p q}\right)$, where the product is taken over all $j, k, p, q=0,1,2$, with $(p, q) \neq(j, k)$. But this determinant is zero if, and only if, $\beta_{j k}=\beta_{p q}$, that is $j+\beta k=p+\beta q$. Since $\beta$ is an irrational number, it follows that $j=p$ and $k=q$, and it is a contradiction. Then $\alpha_{01}=\alpha_{02}=\cdots=\alpha_{22}=0$, which cannot happen, since $P$ is non-constant.

For the general case $m$, observe that each $L_{r}$ is a summation of $(m+1)^{2}-1$ terms, since $\beta_{00}=0$. Then, following the same arguments, we will have a system of dimension $\left((m+1)^{2}-1\right)$, and we will conclude that $\alpha_{j k}=0$, for all possible $j k$ except for $\alpha_{00}$. In this case $P$ would be a constant polynomial, which is a contradiction.

Finally, let $N$ with $L_{N} \neq 0$. Then

$$
H_{n}=\frac{a_{1}}{n !} L_{1}+\frac{a_{2}}{n !} L_{2}+\cdots+\frac{a_{N}}{n !} L_{N}+\cdots+\frac{a_{n}}{n !} L_{n}, n>N .
$$

Applying Lemma 3.1, we have that $\lim _{n \rightarrow \infty}\left|H_{n}\right|=+\infty$.

Using similar ideas from [8], we can prove the following theorem. 
Theorem 3.4. The set $\mathcal{H}$ is algebrable.

Proof: Let $g_{1}(z)=e^{z}$ and $g_{2}(z)=e^{\beta z}$, for all $z \in \mathbb{C}$, where $\beta$ is an irrational number. Since $g_{1}$ and $g_{2}$ are algebraically independent, we have that $\mathcal{A}\left(\left\{g_{1}, g_{2}\right\}\right)$ is isomorphic to $\mathbb{C}\left[z_{1}, z_{2}\right]$, the algebra of all polynomials on two complex variables. Now, if $S=\left\{g_{1}, g_{1} g_{2}, g_{1} g_{2}^{2}, g_{1} g_{2}^{3}, \ldots\right\}$, then $S$ is an infinite minimal system of generators of the algebra $\mathcal{B}=\mathcal{A}(S)$, and $\mathcal{B} \subset \mathcal{A}\left(\left\{g_{1}, g_{2}\right\}\right)$.

Let us fix $f(z)=\frac{1}{z+2}$. We consider $W=\left\{g_{1} \circ f,\left(g_{1} g_{2}\right) \circ f,\left(g_{1} g_{2}^{2}\right) \circ f\right.$, $\left.\left(g_{1} g_{2}^{3}\right) \circ f, \cdots\right\}$. The $W$ is a minimal system of generators for $\mathcal{F}=\{h \circ f$ : $h \in \mathcal{B}\}$. By Theorem 3.3, for each $h \in \mathcal{A}\left(\left\{g_{1}, g_{2}\right\}\right)$, we have that $h \circ f \in \mathcal{H}$. So $\mathcal{F}=\mathcal{A}(W) \subset \mathcal{H} \cup\{0\}$, and therefore $\mathcal{H}$ is algebrable.

We observe that, since $\mathcal{H}$ is closed in $\mathcal{A}(D)$, it follows that $\mathcal{H} \cup\{0\}$ contains a closed infinitely generated algebra.

In the next corollary, we show that not only $\mathcal{H}$ is algebrable, but actually there is an infinite collection of algebra sequences $\left(M_{n}\right)_{n \in \mathbb{N}}$ such that $\mathcal{H}(M)$ is algebrable.

Corollary 3.5. Let $\left(M_{n}\right)_{n \in \mathbb{N}}$ be an algebra sequence such that $M_{n} \leq n !$, for all $n \in \mathbb{N}$. Then $\mathcal{H}(M)$ is algebrable.

Proof: If $M_{n} \leq n !$, then $\mathcal{H} \subset \mathcal{H}(M)$.

Remark 3.6. If $0<\alpha \leq 1$, let $M_{n}:=\alpha^{n} n$ ! for all $n \in \mathbb{N}$. Then $\left(M_{n}\right)_{n \in \mathbb{N}}$ is an algebra sequence such that $M_{n} \leq n !$, for all $n \in \mathbb{N}$.

Acknowledgment: We would like to thank the referee for his/her valuable suggestions that improved this version of the paper.

\section{References}

[1] M. Abtahi and T. G. Honary, On the maximal ideal space of Dales-Davie algebras on infinitely differentiable functions, Bull. London Math. Soc. 39 (2007) 940-948.

[2] M. Abtahi and T. G. Honary, Properties on certain subalgebras of Dales-Davie algebras, Glasgow Math. J. 49 (2007) 225-233.

[3] T. R. Alves, Lineability and algebrability of the set of holomorphic functions with a given domain of existence, Studia Math. 220 (2014), 157-167.

[4] T. R. Alves, Spaceability and algebrability in the theory of domains of existence in Banach spaces, Rev. R. Acad. Cienc. Exactas Fís. Nat. Ser. A Math. RACSAM 109 (2015), 461-470.

[5] R. M. Aron, L. Bernal-González, D. M. Pellegrino and J. B. SeoaneSepúlveda, Lineability. The Search for Linearity in Mathematics, Monographs and Research Notes in Mathematics. Boca Raton, FL, CRC Press, 2016. 
[6] R. M. Aron, V. I. Gurariy and J. B. Seoane-Sepúlveda, Lineability and spaceability of sets of functions on $\mathbb{R}$, Proc. Amer. Math. Soc. 133 (2005) 795-803.

[7] R. M. Aron, D. Pérez-García and J. B. Seoane-Sepúlveda, Algebrability of the set of non-convergent Fourier series, Studia Math. 175 (2006) 83-90.

[8] R. M. Aron and J. B. Seoane-Sepúlveda, Algebrability of the set of everywhere surjective functions on $\mathbb{C}$, Bull. Belg. Math. Soc. 14 (2007) 25-31.

[9] L. Bernal-González and M. O. Cabrera, Lineability criteria, with applications, J. Funct. Anal. 266 (2014) 3997-4025.

[10] H. G. Dales and A. M. Davie, Quasianalytic Banach function algebras, J. Funct. Anal 13 (1973) 28-50.

[11] V. I. Gurariy, Subspaces and bases in spaces of continuous functions, (Russian) Dokl. Akad. Nauk. SSSR 167 (1966) 971-973

[12] V. I. Gurariy, Linear spaces composed of everywhere nondifferentiable functions, C. R. Acad. Bulgare Sci. 44 (1991) 13-16.

[13] T. G. Honary, Relations between Banach function algebras and their uniform closures, Proc. Amer. Math. Soc. 109 (1990) 337-342.

[14] W. P. Johnson, The curious history of Faá di Bruno's formula. Amer. Math. Monthly 109 (2002) 217-234.

Departamento de Matemática

Instituto de Matemática e Estatística

Universidade de São Paulo

Caixa Postal 66281 - CEP : 05315-970

São Paulo, Brazil. e.mails : mllouren@ime.usp.br, danim@ime.usp.br 